\title{
Théologiques
}

\section{Les trente ans de la bioéthique}

\section{Hubert Doucet}

Volume 7, numéro 1, printemps 1999

Les trente ans de la bioéthique

URI : https://id.erudit.org/iderudit/016618ar

DOI : https://doi.org/10.7202/016618ar

Aller au sommaire du numéro

Éditeur(s)

Faculté de théologie de l'Université de Montréal

ISSN

1188-7109 (imprimé)

1492-1413 (numérique)

Découvrir la revue

Citer ce document

Doucet, H. (1999). Les trente ans de la bioéthique. Théologiques, 7(1), 3-7. https://doi.org/10.7202/016618ar d'utilisation que vous pouvez consulter en ligne.

https://apropos.erudit.org/fr/usagers/politique-dutilisation/ 


\title{
Les trente ans de la bioéthique
}

\author{
Hubert DOUCET \\ Faculté de théologie et de médecine \\ Université de Montréal
}

Pourquoi avoir choisi la fin des années 1990 pour marquer les trente ans de la bioéthique? Pourquoi ne pas célébrer ses cinquante ans en rappelant la publication du Code de Nuremberg (1949) qui, à la suite des atrocités nazies, fut un moment déterminant dans le processus de responsabilisation des chercheurs à l'égard des sujets humains? La nécessité du consentement y fut établi et un organisme étranger au monde médical fixa les règles de la pratique. Ou encore, ne serait-il pas plus juste de célébrer ses quarante-cinq ans puisque Joseph Fletcher y publia la première œuvre bioéthique? En effet, c'est en 1954 que parut Morals and Medicine, une démarche, révolutionnaire pour le temps, puisqu'elle se fondait sur l'autonomie du malade plutôt que sur les principes de la morale médicale classique ${ }^{1}$. La même époque vit la découverte du respirateur artificiel, ce qui conduisit un médecin autrichien à écrire au Pape Pie XII pour lui demander conseil en raison des questions difficiles que posait une telle innovation. Selon Stanley Reiser, le Pape lui aurait répondu en confirmant le sérieux de ses questions et en regrettant de ne pouvoir y répondre clairement. Reiser ajoute: "Cet événement où le médecin et le Pape reconnaissent tous deux qu'un problème les dépasse est peut-être un aussi bon moment que n'importe quel autre pour fixer le commencement du mouvement bioéthique ${ }^{2}$." Enfin, pourquoi ne pas avoir retenu le début des années 1960? Ainsi à Seattle, un comité composé de professionnels et de nonprofessionnels sélectionnait les patients qui seraient dialysés.

1 Joseph FletCHER, Morals and Medicine. 2nd ed., Princeton, Princeton University Press, 1979, 243 p.

2 Stanley REISER, «View the Third » dans Hastings Center Report. A Special Supplement, 23 (November-December 1993), p. S14. 
Cette démarche avait pour objectif de tenir compte de tous les éléments nécessaires à une prise de décision éclairée ${ }^{3}$. Albert Jonsen, pour sa part, y voit la naissance de la bioéthique ${ }^{4}$.

Sans nier l'importance de ces divers événements, la fin des années 1960 et le tout début des années 1970 forment une période particulièrement riche de multiples épisodes qui convergeront vers le mouvement bioéthique et donneront naissance au concept lui-même. Dans un mouvement de cette sorte, retenir un fait particulier ne rendrait pas justice à sa dynamique; c'est un ensemble de gestes qui donne sens à ce qui prendra le nom de bioéthique. À partir de 1966 en particulier, s'enchaîne une série d'événements qui va transformer le champ de la biomédecine en raison d'une prise de conscience de professionnels de la santé, de chercheurs, d'universitaires, de politiciens et du public qui considèrent qu'une contradiction profonde habite la médecine moderne : la bienfaisance médicale ne respecte plus la personne du patient. La bioéthique voudra répondre au défi posé par le déve . loppement de la médecine scientifique et technique.

Depuis ce temps, la bioéthique a connu un extraordinaire développement tant aux États-Unis, son lieu d'origine, que dans d'autres pays. Elle est devenue, aux dires de certains, une industrie extraordinairement florissante. Quelques soient les critiques qu'on puisse lui adresser, il faut reconnaître qu'elle occupe une place de choix sur la scène intellectuelle de nos pays. En consa crant un numéro de Théologiques à ces trente ans, l'objectif n'était ni de reprendre la démarche historique ni de présenter les faits saillants qui en ont marqué le développement. La préoccupation était autre. Ce mouvement qui, à son point de départ, était animé du souci du respect de l'autre, en particulier du sujet vulnérable, trente ans plus tard, a-t-il tenu le cap? La démarche proposée ici est donc de nature critique.

À regarder rapidement les titres des divers articles publiés dans ce numéro, l'observateur sera peut-être surpris de n'y retrouver aucun texte de nature historique ni article consacré directe -

R.J. HOWARD et J.S. NAJARIAN, "Organ Transplantation", dans Encyclopedia of Bioethics. New York, The Free Press, 1978, p. $1160-1166$.

4 A.R. JONSEN, "The Birth of Bioethics" Special Supplement, Hastings Center Report, 23 (November-December 1993), p. S1-S4 
ment à la théologie et sa relation à la bioéthique. Quel peut bien être alors le but de Théologiques de consacrer un numéro à la bioéthique? Est-ce un effet de mode? Est-ce le signe que la théologie n'a plus rien de spécifique à contribuer dans le domaine tout en continuant de tirer profit de sa popularité? En ouvrant ses pages aux différents auteurs qui ont été réunis pour l'occasion, Théologiques apporte une contribution essentielle: favoriser la réflexion critique sur ce qu'est devenue cette forme d'éthique. Toutes les études historiques sur l'histoire de la bioéthique témoignent de la place centrale de la théologie dans les premières années du mouvement. C'est avec les théologiens que les chercheurs et les médecins soucieux de l'humanisation de la biomédecine ont d'abord noué le dialogue ${ }^{5}$. Pendant les premiers dix ans de l'entreprise, les théologiens occupèrent une position de premier plan. À partir des années 1980, la théologie s'éclipsa en raison de ce que devenait la bioéthique et de l'impuissance de la théologie de définir sa spécificité. Les théologiens se transformèrent en éthiciens. Le regain d'intérêt pour la théologie est aujourd'hui manifeste. Le souci qui l'habite, les questions qu'elle pose, les affirmations qui sont les siennes retrouvent une grande pertinence, renouant avec les préoccupations premières et fondamentales de l'origine.

En donnant la parole à Susan Sherwin qui réfléchit sur la bioéthique au féminin, Théologiques ouvre un chapitre de l'histoire de la bioéthique qui est à peu près inconnu du public de langue française. Comment la bioéthique, dont le fondement était le souci de l'autre et la justice, a-t-elle intégré la réflexion des femmes sur leur propre condition qui se développait presqu'en même temps? Parce que la bioéthique consistait en une approche nouvelle dans le champ de la morale, la pensée féministe a évité de questionner sa manière d'aborder les questions éthiques. Les deux mouvements étaient, en quelque sorte, de connivence puisqu'ils étaient jeunes et contestaient les idées reçues. Depuis quelques années, des auteures féministes, de même que des penseurs représentant d'autres courants, ont pris conscience du type de pensée qui s'est imposé en bioéthique et qui irait à l'encontre du respect des femmes et de leur condition historique et sociale réelle. Voilà un premier texte qui veut déranger la bioéthique qui s'est institutionnalisée. 
Le deuxième texte est tout aussi dérangeant. La source de sa pensée est plus directement théologique puisque la lecture de la bioéthique qu'il propose est fondée sur la théologie de la libé ration. Si l'acte de naissance de la bioéthique est inséparable d'une demande de justice pour les personnes vulnérables et d'une exigence de protection pour les populations captives, ce souci s'est arrêté aux portes de l'Amérique du Nord et des pays industrialisés. Marcio Fabri dos Anjos aborde la question des inégalités sociales à partir de la situation du Brésil et des pays de l'Amérique latine. Les publications de langue française n'ont à peu près pas consacré d'espace à ce genre de question. En langue anglaise, quelques textes sont récemment parus. À partir de l'option préférentielle pour les pauvres, Marcio Fabri dos Anjos remet en cause la bioé thique telle qu'elle s'est développée au cours des trente dernières années. L'éthique fondée sur les principes universels que sont l'autonomie, la bienfaisance et la justice a exclu de son discours moral la majeure partie de l'humanité.

Si les deux premiers textes abordent des questions habituellement ignorées, le troisième, écrit par Michel Bergeron, traite d'un sujet de grande actualité dans les cercles bioéthiques. En 1998, le Canada et le Québec se sont dotés de nouveaux textes normatifs au plan de l'éthique de la recherche impliquant des sujets humains ${ }^{6}$. Les autorités responsables se montrent de plus de plus exigeantes à l'égard des chercheurs et des institutions locales dans lesquelles ils pratiquent. Les sortes de recherche maintenant poursuivies, le contexte néo-libéral qui place les chercheurs dans des conditions de plus en plus concurrentielles, les scandales qui éclatent parfois peuvent expliquer la tendance à contrôler davantage les chercheurs. Après les comités d'éthique qui ont la responsabilité d'évaluer les projets des chercheurs, voilà que ces mêmes comités se voient confiés la responsabilité de faire la surveillance continue de ces projets. Ces comités deviendront-ils la police de la recherche? $\grave{A}$ partir d'un problème concret et ponctuel, Michel Bergeron nous permet de saisir les limites qui confrontent aujourd'hui l'idéal bioéthique de la fin des années 1960.

6 Énoncé de politique des trois Conseils, Éthique de la recherche avec des êtres humains, 1998; MSSS, Plan d'action ministériel en éthique de la recherche et en intégrité scientifique, 1998. 
Le quatrième texte du cahier est intitulé «Misères et grandeurs de la bioéthique ». Il est écrit par Guy Durand. Depuis vingt ans maintenant, ce dernier a été l'animateur de la bioéthique à l'Université de Montréal. S'il fut d'abord professeur de théologie morale à la Faculté de théologie, la bioéthique est peu à peu devenue son champ d'expertise. Invité à être professeur d'éthique médicale à la Faculté de médecine et membre de nom. breux comités d'éthique dans les hôpitaux universitaires, il a créé les différents programmes de bioéthique offerts présentement à l'Université. Ayant ainsi œuvré sur le terrain, dans le contexte québécois, l'auteur est particulièrement bien situé pour éclairer le passé et le présent de la bioéthique. Après toutes ces années, quel jugement porte-t-il?

En lisant ces textes, j'espère que la lectrice ou le lecteur peu familier avec la bioéthique y découvrira un champ qui, en raison de l'importance des questions qu'il étudie et des attentes qu'il suscite, mérite d'être pris au sérieux et soumis, pour le succès de sa mission propre, à une critique permanente. Quant aux spécialistes, puissent-ils y trouver l'occasion d'élargir le champ de leurs préoccupations en entrant en dialogue avec des types de questionnement que les exigences du quotidien ne leur donnent pas toujours la chance d'aborder. Si ce numéro de Théologiques favorisait l'élargissement des perspectives et la poursuite du dialogue, il aurait largement atteint son objectif. 